Supplement of Biogeosciences, 18, 5699-5717, 2021 https://doi.org/10.5194/bg-18-5699-2021-supplement (c) Author(s) 2021. CC BY 4.0 License.

(c) (1)

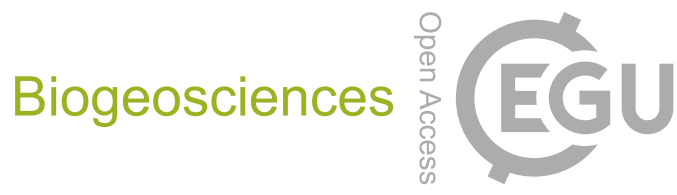

Supplement of

\title{
Influence of atmospheric deposition on biogeochemical cycles in an oligotrophic ocean system
}

France Van Wambeke et al.

Correspondence to: France Van Wambeke (france.van-wambeke@mio.osupytheas.fr) and Cécile Guieu (guieu@imev-mer.fr)

The copyright of individual parts of the supplement might differ from the article licence. 
Page 2: Figure S1

Page 3: Figure S2

Page 4: Figure S3

Page 5: Enrichment experiment and Fig S4

Page 6: Table S1

Page 7: Table S2 
Figure S1: Vertical distribution of heterotrophic prokaryotic production (BP), particulate primary production (PP), and abundances of heterotrophic prokaryotes (hprok), Synechococcus-like cells (syn), eukaryotic picophytoplankton (pico euk) and nanophytoplankton (nano euk) at the ION site. Casts numbered the date of their sampling before (blue profiles) and after (grey profiles) the rain sampled onboard.
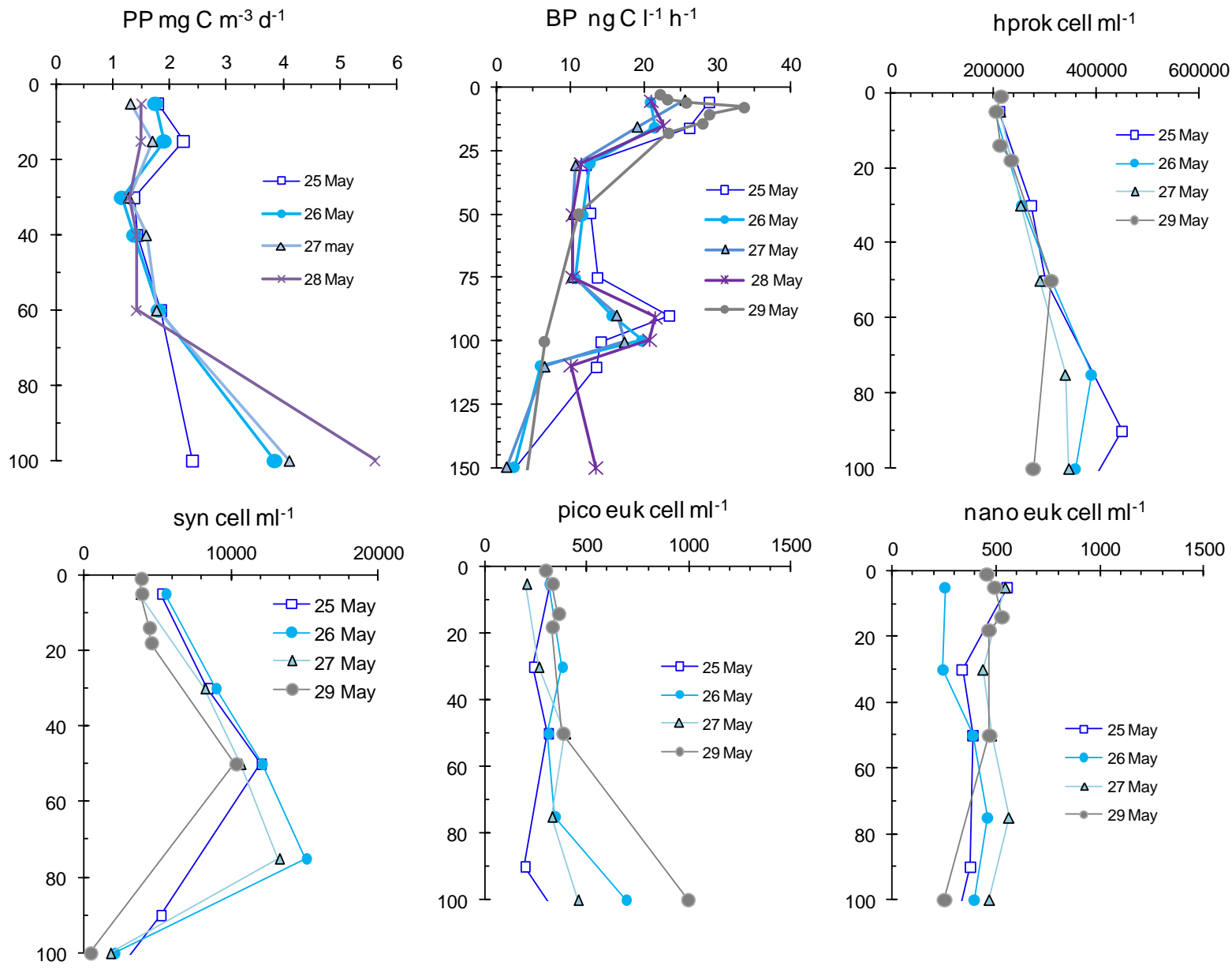
Figure S2: Vertical distribution of heterotrophic prokaryotic production (BP), particulate primary production (PP), and in vivo fluorescence profiles at the FAST site. Stations numbered in days before (blue profiles) and after (grey profiles) the rain event.
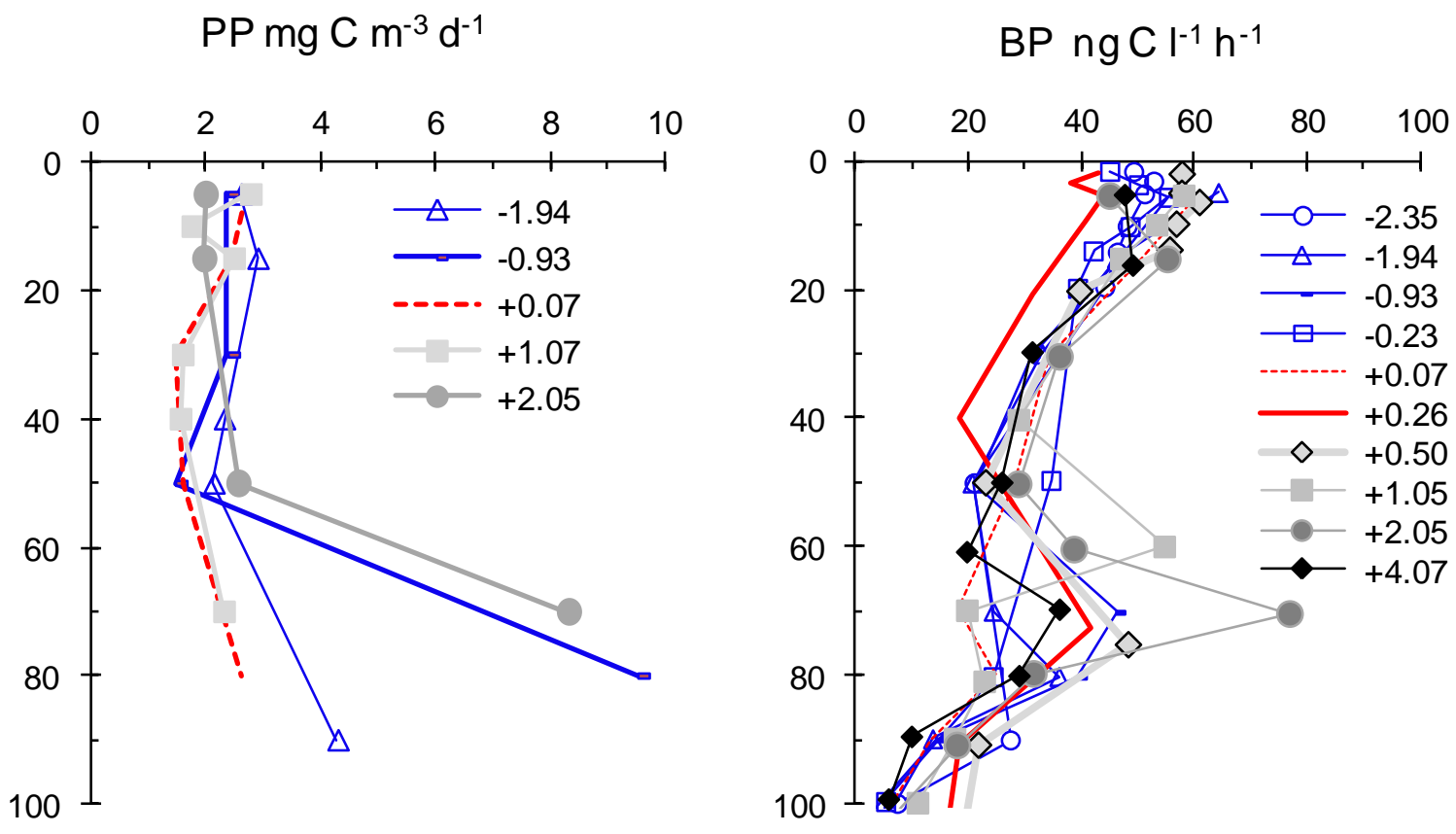

in vivo fluorescence $\mu \mathrm{g} \mathrm{I}^{-1}$

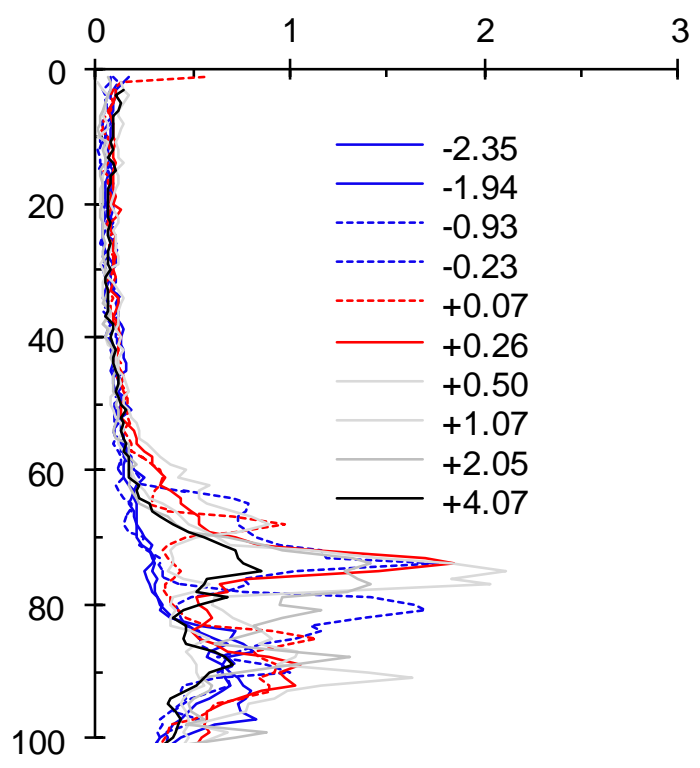


Figure S3. Vertical distribution of abundances for heterotrophic prokaryotes (hprok), heterotrophic nanoflagellates (HNF), Synechococcus-like cells (syn), Prochlorococcus (proc), eukaryotic picophytoplankton (pico euk) and nanophytoplankton (nano euk) at the FAST site. Stations numbered in days before (blue profiles) and after (grey profiles) the rain event sampled on board.
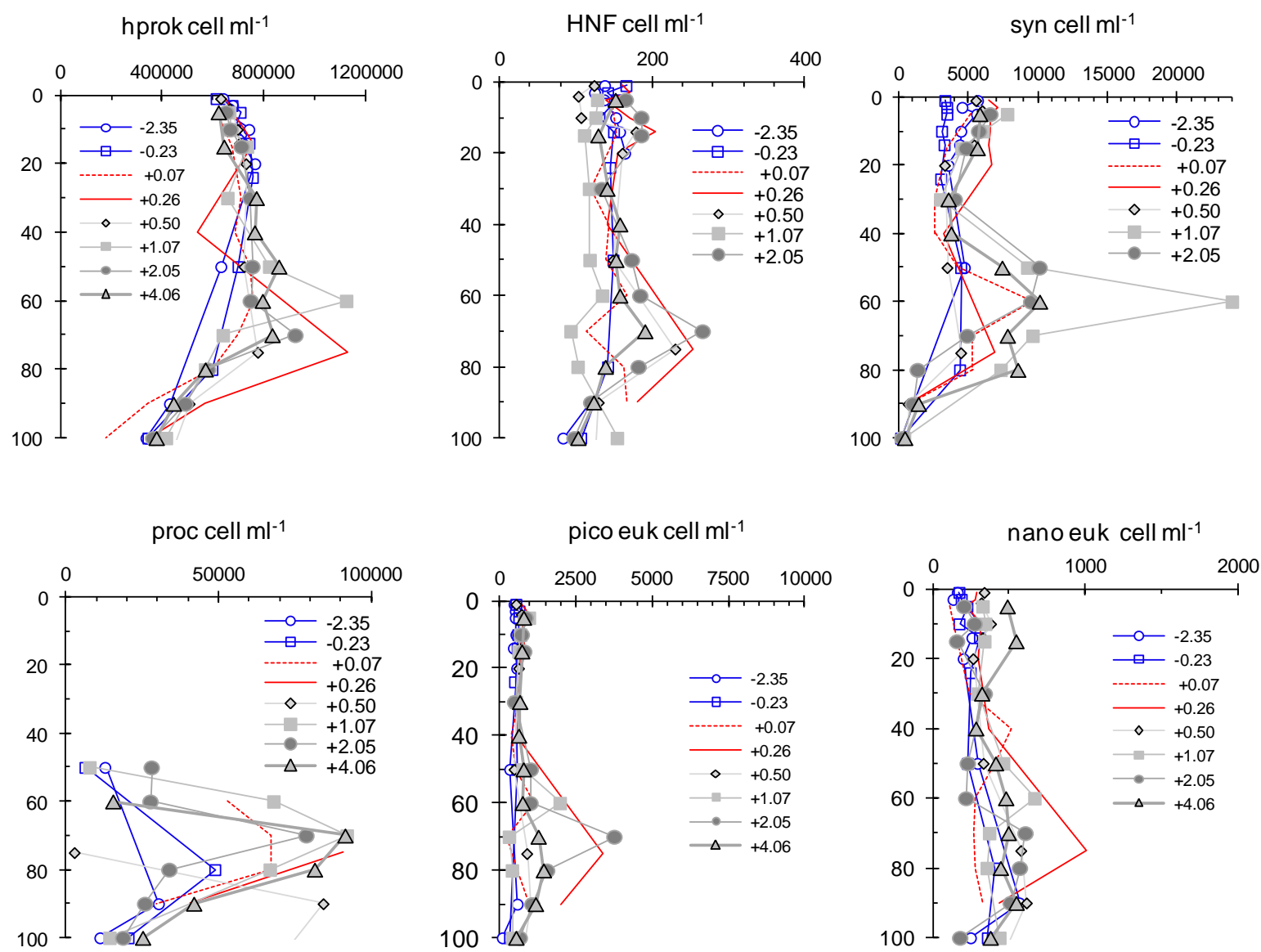


\section{Enrichment experiments}

At the three sites, enrichment experiments were performed using seawater from $5 \mathrm{~m}$ depth to assess factors limiting BP in the surface mixed layer. The sampling of seawater for these experiments [FAST (June 2, 22:00), TYR (May 16, 20:00) and ION (May 25, 20:00)] was done before the rain events occurring at the FAST and ION sites. Eight series of triplicate 60 $\mathrm{mL}$ polycarbonate bottles were filled with unfiltered seawater and amended as follows: $\mathrm{C}: \mathrm{no}$ enrichment, $\mathrm{N}:+1 \mu \mathrm{M}$ NO3 + $1 \mu \mathrm{M}$ NH4; P: + $0.2 \mu \mathrm{M}$ DIP; G: + $10 \mu \mathrm{M}$ C-glucose; NP: N + $\mathrm{P} ; \mathrm{NG}: \mathrm{N}+\mathrm{G} ; \mathrm{PG}: \mathrm{P}+\mathrm{G} ; \mathrm{NPG}: \mathrm{N}+\mathrm{P}+\mathrm{G}$. After $48 \mathrm{~h}$ incubation in the dark at in situ temperature, BP was determined in the 24 bottles (as described in M\&M section).

At the TYR site, BP was significantly stimulated only after addition of 2 major elements, with a greater response with PN and NPG combinations (Fig. S4). At the ION site, BP was primarily limited by $\mathrm{P}$ availability, as only combinations with $\mathrm{P}$ (single or in combination with other elements), stimulated BP whereas all other combinations of enrichment did not stimulate BP significantly compared to the control. At the FAST site, BP was primarily limited by $\mathrm{N}$ availability, as all combinations with $\mathrm{N}$, single or in combination with other elements, stimulated BP. However at this site it is likely that BP was also co-limited by 2 elements, as G addition alone also stimulated BP. Note that the PN combination has induced a higher stimulation of BP than other double combinations (NG or PG).

Figure S4. Results of the enrichment experiment. BP reached after $36 \mathrm{~h}$ of enrichment in the dark. C: control unamended, $\mathrm{P}:+\mathrm{DIP}, \mathrm{N}:+\mathrm{NO} 3+\mathrm{NH} 4, \mathrm{G}:+$ glucose, and combinations of these elements in PN, NG, PG and NPG. Stars show significantly different BP compared to the unamended control .

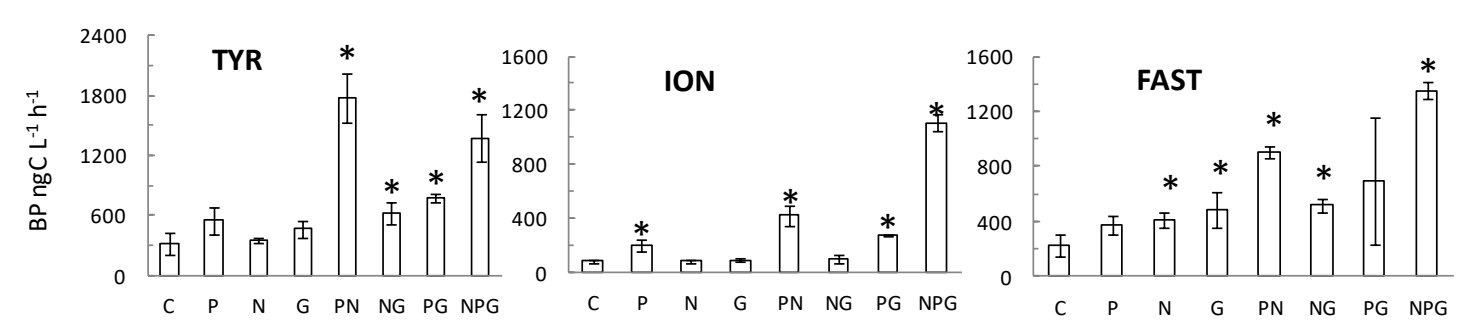


Table S1: NO3 ranges within the surface mixed layer (ML) and the base of the nitrate depleted layer below (NDLb) and advective fluxes when measurable. CTDs at the FAST sit are chronologically indexed in days before (negative index) and after (positive index) the occurrence of the rain event collected on board. no lwcc: no data because at these stations concentrations were determined only by classical analysis and were under its detection limits (50 nM), na : not available. From comparison of concentrations, we identified four groups of stations: group 1: poor nitrates in ML and NDLb $(<50 \mathrm{nM})$; weak differences $(<15 \mathrm{nM})$; group 2: moderate nitrates in ML and NDLb $(50 \mathrm{nM}$ $<\mathrm{NO3}<80 \mathrm{nM}$ ); weak differences $(<20 \mathrm{nM}$ ); group 3: high nitrate in ML and NDLb (NO3 > $80 \mathrm{nM}$ ); weak positive differences $(<20 \mathrm{nM})$ and group 4:high nitrate in ML and moderate to high in NDLb, large positive differences (> $20 \mathrm{nM}$ ). MLD: mixed layer depth. sd of fluxes were estimated using propagation of errors on $\mathrm{NO} 3_{\mathrm{ML}}$, NO3 ${ }_{\mathrm{NDLb}}$ and $\mathrm{dMLD} / \mathrm{dt}$.

\begin{tabular}{|c|c|c|c|c|c|c|c|c|c|c|c|c|c|}
\hline & \multirow[b]{2}{*}{ Date, local time } & \multirow[b]{2}{*}{$\begin{array}{c}\text { MLD } \\
\mathrm{m}\end{array}$} & \multirow[b]{2}{*}{$\begin{array}{c}\text { base NDLb } \\
\mathrm{m}\end{array}$} & \multicolumn{3}{|c|}{ NO3 in ML } & \multicolumn{3}{|c|}{$\mathrm{NO} 3$ in NDLb } & \multirow{2}{*}{$\begin{array}{c}\text { difference } \\
\mathrm{nM} \\
\end{array}$} & \multicolumn{2}{|c|}{ 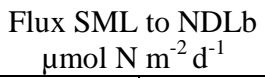 } & \multirow[b]{2}{*}{ Station group } \\
\hline & & & & $\begin{array}{c}\text { Mean } \\
\mathrm{nM}\end{array}$ & $\mathrm{sd} \mathrm{nM}$ & $\mathrm{n}$ & Mean nM & $\mathrm{sd} n \mathrm{M}$ & $\mathrm{n}$ & & Mean & sd & \\
\hline ST 1 & 12/05/2017 12:26 & 21 & 52 & no lwec & & & no lwec & & & & na & & na \\
\hline ST2 & 13/05/2017 07:40 & 21 & 60 & no lwec & & & no lwce & & & & na & & na \\
\hline ST3 & 14/05/2017 12:05 & 11 & 73 & no lwec & & & no lwce & & & & na & & na \\
\hline ST4 & 15/05/2017 07:09 & 15 & 55 & no lwec & & & no lwce & & & & na & & na \\
\hline ST5 & 16/05/2017 04:04 & 9 & 66 & 29 & na & 1 & 17 & 8 & 2 & 13 & na & & 1 \\
\hline ST TYR 17 May & 17/05/2017 09:36 & 9 & 69 & 14 & 0 & 2 & 19 & 6 & 4 & -5 & na & & 1 \\
\hline ST6 & 22/05/2017 05:48 & 18 & 71 & 9 & na & 1 & 9 & 0 & 2 & 0 & na & & 1 \\
\hline ST7 & 23/05/2017 21:11 & 18 & 79 & 9 & na & 1 & 9 & 0 & 2 & 0 & na & & 1 \\
\hline ST ION 25 May & 25/05/2017 05:28 & 14 & 86 & 14 & 5 & 2 & 14 & 6 & 6 & 0 & na & & 1 \\
\hline ST ION 27 May & 27/05/2017 08:24 & 18 & 93 & 127 & 31 & 6 & 103 & 12 & 2 & 24 & 45 & 52 & 4 \\
\hline ST ION 29 May & 29/05/2017 09:19 & 16 & 88 & 92 & 17 & 6 & 67 & na & 1 & 25 & -25 & 21 & 4 \\
\hline ST8 & $30 / 05 / 201704: 48$ & 14 & 79 & 60 & na & 1 & 68 & 12 & 3 & -8 & na & & 2 \\
\hline ST9 & 01/06/2017 21:15 & 7 & 72 & 117 & na & 1 & 106 & 15 & 2 & 11 & na & & 3 \\
\hline ST FAST -2.3 & 02/06/2017 19:16 & 9 & 80 & 79 & 4 & 3 & 74 & 15 & 3 & 6 & 0 & 0 & 2 \\
\hline ST FAST -1.5 & 03/06/2017 15:30 & 13 & 78 & 59 & 3 & 2 & 46 & 5 & 5 & 13 & 62 & 34 & 2 \\
\hline ST FAST -0.25 & 04/06/2017 20:57 & 12 & 77 & 56 & 4 & 3 & 54 & 14 & 3 & 2 & -2 & 9 & 2 \\
\hline ST FAST +024 & 05/06/2017 08:54 & 16 & 77 & 93 & 15 & 5 & 51 & 7 & 3 & 42 & 337 & 173 & 4 \\
\hline ST FAST +0.53 & 05/06/2017 15:50 & 16 & 82 & 70 & 5 & 4 & 50 & na & 1 & 20 & 0 & 69 & 4 \\
\hline ST FAST +1.05 & 06/06/2017 04:23 & 19 & 85 & 39 & 3 & 4 & 45 & 9 & 2 & -6 & -34 & 46 & 1 \\
\hline ST FAST + 2.11 & 07/06/2017 05:38 & 15 & 59 & 9 & 0 & 3 & 19 & 3 & 2 & -10 & 38 & 15 & 1 \\
\hline ST10 & 08/06/2017 06:55 & 19 & 62 & 120 & na & 1 & 58 & 15 & 3 & 62 & na & & 4 \\
\hline ST FAST +3.8 & 08/06/2017 22:09 & 17 & 73 & 135 & 7 & 3 & 116 & 22 & 3 & 20 & 22 & 28 & 4 \\
\hline
\end{tabular}


Table S2. Biological stocks and fluxes integrated over the mixed layer (a), the euphotic zone (b) and $200 \mathrm{~m}$ (c), along with atmospheric dry deposition during the occupation of the ION and FAST sites. Time CTD cast is the local time at the beginning of the CTD cast. For atmospheric dry deposition, start and end shows the period during which PILS data were averaged to compute daily DIN (NO3+NH4) depositions fluxes. PP: Primary production, BP: heterotrophic prokaryotic production, LAP: In situ leucine aminopeptidase hydrolysis rates, N2fix: $\mathrm{N}_{2}$ fixations rates.

\begin{tabular}{|c|c|c|c|c|c|c|c|c|c|c|c|c|}
\hline & \multirow[b]{2}{*}{$\begin{array}{l}\text { time CTD } \\
\text { cast }\end{array}$} & \multicolumn{6}{|c|}{ biological flux } & \multicolumn{2}{|c|}{ stocks } & \multicolumn{3}{|c|}{ dry deposition } \\
\hline & & $\begin{array}{l}\mathrm{PP}^{\mathrm{b}} \\
\mathrm{mg} \mathrm{C} \mathrm{m}^{-2} \mathrm{~d}^{-1}\end{array}$ & $\begin{array}{l}\mathrm{BP}^{\mathrm{c}} \\
\mathrm{mg} \mathrm{C} \mathrm{m}^{-2} \mathrm{~d}^{-1}\end{array}$ & 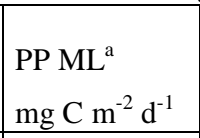 & $\begin{array}{l}\mathrm{BP} \mathrm{ML}^{\mathrm{a}} \\
\mathrm{mg} \mathrm{C} \mathrm{m}^{-2} \mathrm{~d}^{-1}\end{array}$ & $\begin{array}{l}\text { LAP ML }^{\mathrm{a}} \\
\mu \mathrm{mol} \mathrm{N} \mathrm{m}{ }^{-2} \mathrm{~d}^{-1}\end{array}$ & $\begin{array}{l}\text { N2fix } \mathrm{ML}^{\mathrm{a}} \\
\mu \mathrm{mol} \mathrm{N} \mathrm{m}^{-2} \mathrm{~d}^{-1}\end{array}$ & $\begin{array}{l}\text { NO3 stocks } \\
\mathrm{ML}^{\mathrm{a}} \\
\mu \mathrm{mol} \mathrm{N} \mathrm{m}{ }^{-2}\end{array}$ & $\begin{array}{l}\text { DIP stocks } \\
\mathrm{ML}^{\mathrm{a}} \\
\mu \mathrm{mol} \mathrm{P} \mathrm{m}^{-2}\end{array}$ & start & end & $\begin{array}{l}\text { DIN } \\
\mu \text { mole } \mathrm{N} \mathrm{m}^{-2} \mathrm{~d}^{-1}\end{array}$ \\
\hline TYR 17 May & $17 / 5 / 174: 58$ & & 5.6 & 13.7 & 5.6 & 17.2 & 3.9 & 127 & 64 & $17 / 5 / 172: 54$ & 17/5/17 4:58 & 23.2 \\
\hline TYR 18 May & $18 / 5 / 174: 57$ & & 6.0 & 12.0 & 6.0 & & & & & 17/5/17 4:58 & 18/5/17 4:57 & 25.1 \\
\hline TYR 19 May & $19 / 5 / 174: 53$ & & 6.1 & 13.9 & 6.1 & & & & & 18/5/17 4:57 & 19/5/17 4:53 & 26.9 \\
\hline TYR 20 May & $20 / 5 / 174: 45$ & & 14.5 & 46.1 & 14.5 & & & & & 19/5/17 4:53 & 20/5/17 4:45 & 37.9 \\
\hline ION 25 May & $25 / 5 / 174: 34$ & 188 & 56.1 & 19.7 & 7.5 & 27.8 & 6.1 & 195 & 142 & 24/5/17 18:02 & 25/5/17 4:34 & 24.1 \\
\hline ION 26 May & 26/5/17 4:25 & 207 & 44.7 & 26.9 & 8.0 & & & & & 25/5/17 4:34 & $26 / 5 / 174: 25$ & 27.8 \\
\hline ION 27 May & 27/5/17 4:26 & 210 & 43.9 & 19.9 & 8.0 & & 5.1 & 2113 & 109 & $26 / 5 / 174: 25$ & $27 / 5 / 174: 26$ & 32.6 \\
\hline ION 28 May & 28/5/17 4:21 & 226 & & 31.3 & 10.7 & & & & & 27/5/17 4:26 & 28/5/17 4:21 & 33.8 \\
\hline ION 29 May & 29/5/17 9:19 & & 45.2 & & 10.3 & & 6.3 & 1477 & 167 & 28/5/17 4:21 & 29/5/17 9:19 & 29.2 \\
\hline FAST-2.32 & 2/6/17 18:37 & & 87.8 & & 13.2 & 31.1 & & 715 & 99 & 2/6/17 20:24 & 2/6/17 18:37 & \\
\hline FAST-1,48 & $3 / 6 / 174: 32$ & 257 & 81.2 & 37.8 & 19.9 & & 5.9 & 751 & 162 & 2/6/17 18:37 & $3 / 6 / 174: 32$ & 42.5 \\
\hline FAST-0,93 & $4 / 6 / 174: 40$ & 274 & 87.9 & 28.5 & 15.6 & & 7.1 & & & $3 / 6 / 174: 32$ & $4 / 6 / 174: 40$ & 38.3 \\
\hline FAST- 0,25 & $4 / 6 / 1721: 27$ & & 88.9 & & 12.4 & & & 665 & 137 & $4 / 6 / 174: 40$ & $4 / 6 / 1721: 27$ & 61.2 \\
\hline FAST 0.07 & $5 / 6 / 174: 44$ & 164 & 80.8 & 29.3 & 15.6 & & 6.4 & & & 4/6/17 21:27 & $5 / 6 / 174: 44$ & 39.6 \\
\hline FAST 0,24 & $5 / 6 / 179: 24$ & & 92.7 & & 14.6 & 47.2 & & 1520 & 136 & $5 / 6 / 174: 44$ & $5 / 6 / 179: 24$ & \\
\hline FAST 0,53 & $5 / 6 / 1714: 59$ & & 113.3 & & 19.4 & 52.8 & & 1113 & 210 & $5 / 6 / 179: 24$ & $5 / 6 / 1714: 59$ & 12.9 \\
\hline FAST 1,05 & $6 / 6 / 174: 51$ & 140 & 99.2 & 33.4 & 18.6 & 54.8 & 8.3 & 752 & 281 & $5 / 6 / 17$ 14:59 & $6 / 6 / 174: 51$ & \\
\hline FAST 2.11 & $7 / 6 / 174: 17$ & 218 & 104.1 & 31.8 & 18.7 & 35.1 & 8.1 & 177 & 194 & $6 / 6 / 174: 51$ & $7 / 6 / 174: 17$ & 19.8 \\
\hline FAST 3.8 & $9 / 6 / 174: 40$ & & 82.9 & & 21.9 & 34.5 & & 2314 & 125 & 8/6/17 21:06 & 9/6/17 0:16 & 23.9 \\
\hline
\end{tabular}

